\title{
DE CVn: A Bright, Eclipsing Red Dwarf - White Dwarf Binary
}

E. J. M. van den Besselaar ${ }^{1}$, R. Greimel ${ }^{2}$, L. Morales-Rueda ${ }^{1}$, G. Nelemans ${ }^{1}$, J. R. Thorstensen ${ }^{3}$, T. R. Marsh ${ }^{4}$, V. S. Dhillon ${ }^{5}$, R. M. Robb ${ }^{6}$, D. D. Balam ${ }^{6}$, E. W. Guenther ${ }^{7}$, J. Kemp ${ }^{8}$, T. Augusteijn ${ }^{9}$ and P. J. Groot ${ }^{1}$

${ }^{1}$ Department of Astrophysics, IMAPP, Radboud University Nijmegen, PO Box 9010, 6500 GL

Nijmegen, The Netherlands email: [besselaar;lmr;nelemans;pgroot]@astro.ru.nl

${ }^{2}$ Isaac Newton Group of Telescopes, Apartado de correos 321, E-38700 Santa Cruz de la

Palma, Spain email: greimel@ing.iac.es

${ }^{3}$ Department of Physics and Astronomy, Dartmouth College, 6127 Wilder Laboratory

Hanover, NH 03755, USA email: thorsten@partita.dartmouth.edu

${ }^{4}$ Department of Physics, University of Warwick, Coventry CV4 7AL, UK email: t.r.marsh@warwick.ac.uk

${ }^{5}$ Department of Physics and Astronomy, University of Sheffield, Sheffield S3 7RH, UK email: vik.dhillon@sheffield.ac.uk

${ }^{6}$ Department of Physics and Astronomy, University of Victoria, Victoria, BC, V8W 3P6, Canada email: robb@uvic.ca; cosmos@uvvm.uvic.ca

${ }^{7}$ Thüringer Landessternwarte Tautenburg, Sternwarte 5, D-07778 Tautenburg, Germany email: guenther@tls-tautenburg.de

${ }^{8}$ Joint Astronomy Centre 660 N. A'ohoku Place University Park Hilo, Hawaii 96720, USA email: j.kemp@jach.hawaii.edu

${ }^{9}$ Nordic Optical Telescope, Apartado 474, E-38700 Santa Cruz de La Palma, Spain email: tau@not.iac.es

\begin{abstract}
DE CVn is a relatively unstudied eclipsing binary where one of the components is an $\mathrm{M}$ dwarf and the other is a white dwarf. Its brightness makes it an ideal system for a detailed study in the context of common-envelope evolution of a detached white dwarf - red dwarf binary with a relatively short orbital period ( $\sim 8.7$ hours). We present a detailed study of the basic parameters (e.g. orbital period, components' masses and spectral types) for this system from photometric and spectroscopic studies. The eclipses observed during several photometric observing runs were used to derive the ephemeris. We have used spectroscopic data to derive the radial velocity variations of the emission lines and these are used to determine the components' masses and the orbital separation. The secondary component in DE CVn is an M3 main-sequence star and the primary star, which only contributes to the blue continuum, is a cool white dwarf with a temperature of $\sim 8000 \mathrm{~K}$. From the photometry and spectroscopy together, we have set a limit on the binary inclination. This system is a post-common-envelope system where the progenitor of the present day white dwarf was a low-mass star $\left(\mathrm{M} \leqslant 2 M_{\odot}\right)$. The time before DE CVn becomes a semi-detached system is longer than the Hubble time.
\end{abstract}

Keywords. Stars: individual (DE CVn), Binaries: close, Binaries: eclipsing, Stars: late-type, white dwarf, Stars: fundamental parameters

\section{Introduction}

DE CVn is a high proper motion object $\left(-0^{\prime \prime} .198 \pm 0^{\prime \prime} .002\right.$ in $\mathrm{RA},-0^{\prime \prime} .178 \pm 0^{\prime \prime} .003$ in Dec) which was first discovered as an X-ray source by ROSAT (Voges et al. 1999). It is a 


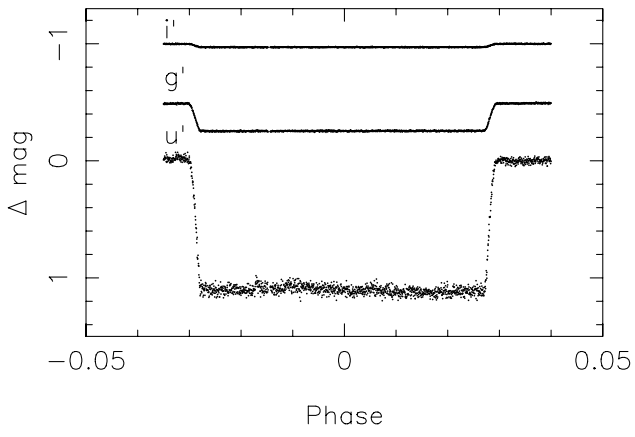

Figure 1. Primary eclipse observed with ULTRACAM.

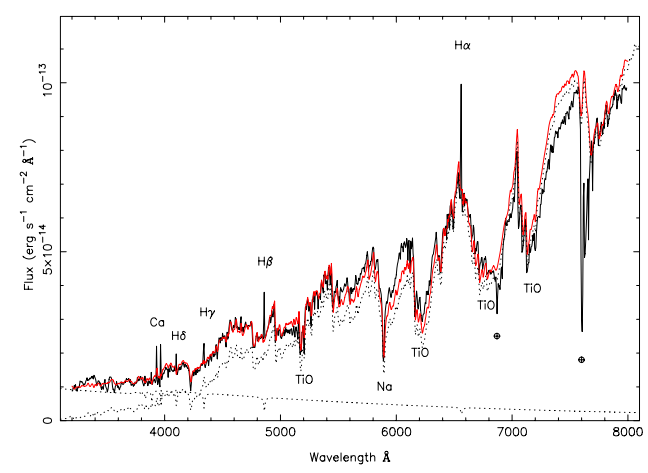

Figure 2. A combined ISIS spectrum of DE CVn (black line) together with the best model (grey/red). See Table 1 for information on the model.

detached white dwarf - red dwarf (WD+RD) binary with a relatively short ( $\sim 8.7$ hours) orbital period. All close WD+RD binaries must have gone through a common-envelope (CE) phase during their evolution. DE CVn's brightness and the presence of eclipses makes this system ideal for a more detailed study. Our aim is to derive its system parameters from a study of photometric and spectroscopic observations and ultimately to set limits on the physics of the CE phase.

\section{Observations}

We obtained photometry and spectroscopy of DE CVn on a number of telescopes and epochs. Simultaneous photometry was obtained in $u^{\prime}, g^{\prime}$ and $i^{\prime}$ bands with ULTRACAM on the WHT on May 24th, 2003. Additional photometry was obtained with the automatic 0.5 -meter telescope of the Climenhage Observatory in Victoria, Canada $(R, V$ and clear filters), with the 1.8-meter telescope of the Dominion Astrophysical Observatory ( $B$ filter) and with the 1.3-meter telescope of the Michigan-Dartmouth-MIT Observatory (MDM) in Arizona ( $B, B G 38$ filters) over a period of 10 years. The main spectroscopic observations are echelle observations with the 2-m telescope of the Thüringer Landessternwarte 'Karl Schwarzschild' in Tautenburg and long-slit spectroscopy with the MDM and WHT telescopes.

\section{Photometry}

By combining our photometry and the published times of mid-eclipse from Robb \& Greimel (1997) and Tas et al. (2004), we obtain a new, more accurate ephemeris:

$$
H J D_{\min }=2452784.55337(2)+0.36413945(4) \times E
$$

with the uncertainty on the last digits in parentheses. Figure 1 shows the ULTRACAM photometry of the eclipse of DE CVn on the night of May 24th in 2003.

The eclipse depths in $u^{\prime}, g^{\prime}$ and $i^{\prime}$ are $1.11 \pm 0.04,0.235 \pm 0.004$ and $0.028 \pm 0.004$ magnitudes respectively.

\section{Spectroscopy}

We have used the low-resolution spectra obtained with ISIS on the WHT to derive the composition of DE CVn. The WD atmospheres were kindly provided by P. Bergeron 


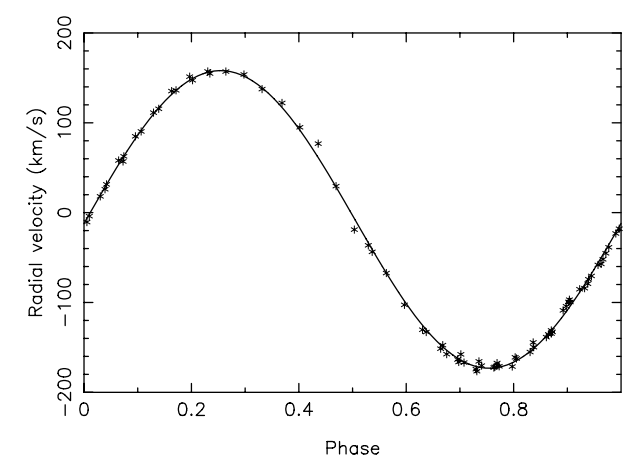

Figure 3. Radial velocity measurements for the $\mathrm{H} \alpha$ lines. The solid line is the best fit to these velocities. The uncertainties on the points are smaller than the symbols.

(with $1500 \mathrm{~K}<\mathrm{T}<17000 \mathrm{~K}$ ). RD templates (M0V - M6V) have been obtained from Pickles (1998). Single templates are first scaled to $10 \mathrm{pc}$, then added and fitted to the observed spectrum, including a distance offset. The combination with the lowest $\chi^{2}$ is taken as the best fit and is shown in Figure 2. From the echelle spectroscopy of the $\mathrm{H} \alpha$ line we derive the radial velocity curve of the RD as shown in Figure 3. The semi-amplitude is $166 \pm 4 \mathrm{~km} \mathrm{~s}^{-1}$.

\section{Conclusion}

The results of our photometric and spectroscopic analysis are listed in Table 1. DE CVn is an eclipsing binary consisting of a cool WD and an M3 main-sequence star that must have experienced a CE phase. From evolutionary tracks we derive a WD progenitor mass of $\mathrm{M} \leqslant 2 M_{\odot}$. The time remaining before the system becomes semi-detached is $1.7 \times 10^{10}$ years. These kinds of systems will not contribute to the current sample of Cataclysmic Variables, unless the loss of angular momentum in the current detached phase is much higher than that given by magnetic braking alone.

Table 1. System parameters of DE CVn

\begin{tabular}{ll|ll} 
Parameter & Value & Parameter & Value \\
\hline WD temperature & $8000 \pm 2000 \mathrm{~K}$ & WD mass & $0.54 \pm 0.04 M_{\odot}$ \\
WD $\log g$ & 7.5 & WD radius & $0.0132 \pm 0.0006 R_{\odot}$ \\
RD spectral type & $\mathrm{M} 3 \mathrm{~V}$ & RD mass & $0.40 \pm 0.05 M_{\odot}$ \\
Semi-amplitude & $166 \pm 4 \mathrm{~km} \mathrm{~s}^{-1}$ & RD radius & $0.40 \pm 0.04 R_{\odot}$ \\
Orbital separation & $2.10 \pm 0.06 R_{\odot}$ & Inclination & $\geqslant 82^{\circ}$ \\
Distance & $26 \pm 3 \mathrm{pc}$ & &
\end{tabular}

\section{Acknowledgements}

We thank Pierre Bergeron for making his cool white dwarf models available to us.

EvdB, LMR and PJG are supported by NWO-VIDI grant 639.042.201 to P.J. Groot. GN is supported by NWO-VENI grant 638.041.405 to G. Nelemans. JRT thanks the U.S. National Science Foundation for support through grants AST-9987334 and AST-0307413. TRM was supported by a PPARC Senior Fellowship during the course of this work.

ULTRACAM is supported by PPARC grants PP/D002370/1 and PPA/G/S/2003/ 00058. The William Herschel Telescope is part of the Isaac Newton Group of Telescopes, operated on the island of La Palma by the Instítuto de Astrofísica de Canarias on behalf of 
the British PPARC and the Dutch NWO. We acknowledge the use of the $0.5-\mathrm{m}$ telescope of the Cimenhage Observatory and the 1.8-m telescope of the Dominion Astrophysical Observatory located in Victoria, Canada. We acknowledge the use of the 1.3-m telescope and the 2.4-m Hiltner Telescope of the Michigan-Dartmouth-MIT observatory in Arizona and the 2-m Alfred-Jensch-Teleskop at the Thüringer Landessternwarte.

\section{References}

Pickles, A.J. 1998, PASP, 110, 863

Robb, R.M. \& Greimel, R. 1997, Informational Bulletin on Variable Stars, 4486, 1

Tas, G., Sipahi, E., Dal, H.A., et al. 2004, Information Bulletin on Variable Stars, 5548, 1

Voges, W., Aschenback, B., Boller, T., et al. 1999, A\&્A, 349, 389 